\title{
Research Letter \\ Patterning of Gold Nanoparticles on DNA Self-Assembled Scaffolds
}

\author{
Immensee Cheng, ${ }^{1}$ Bryan Wei, ${ }^{1}$ Xunyun Zhang, ${ }^{1}$ Yongjian Wang, ${ }^{2}$ and Yongli Mi ${ }^{1}$ \\ ${ }^{1}$ Department of Chemical Engineering, School of Engineering, The Hong Kong University of Science and Technology, \\ Clear Water Bay, Kowloon, Hong Kong \\ ${ }^{2}$ Department of Physics, School of Science, The Hong Kong University of Science and Technology, Clear Water Bay, \\ Kowloon, Hong Kong \\ Correspondence should be addressed to Yongli Mi, keymix@ust.hk
}

Received 21 November 2007; Accepted 4 January 2008

Recommended by Chuan-Jian Zhong

We report a method of patterning the 1D and 2D arrays of gold nanoparticles on the DNA self-assembled scaffolds. The $5 \mathrm{~nm}$ gold nanoparticles were well positioned at the center of each $4 \times 4$ tile motif of the DNA scaffold. The precisely located gold particles can form 1D and 2D arrays. This controllable scaffolding technology may become a promising tool for nanoscaled fabrication of electronics and photonic devices.

Copyright (C) 2008 Immensee Cheng et al. This is an open access article distributed under the Creative Commons Attribution License, which permits unrestricted use, distribution, and reproduction in any medium, provided the original work is properly cited.

\section{INTRODUCTION}

In recent years, people have shown tremendous interest in developing electronic, photonic, and spintronic devices with nanoparticles (NPs). Since the top-down approach requires expensive equipment and expertise, the bottom-up self-assembly approach has the advantage of massive parallelism. The challenge of the bottom-up assembly is to build well-defined structures with considerable rigidity to scaffold NP assemblies. Lately, substantial attention has been drawn to self-assembled DNA suprastructures because DNA and DNA-based nanostructures are programmable and rigid enough. Meanwhile, DNA can be easily grafted to the surface of the NPs, so that the self-assembled DNA suprastructures can be used as templates for patterning NPs $[1,2]$.

Although the concept of using DNA to scaffold NPs was first proposed by Robinson and Seeman about two decades ago [3], the development of DNA self-assembly, as scaffolds for functional nanomaterials, is still in its early stages. To follow the idea of DNA-based nanopatterning, researchers have developed a variety of motifs for DNA self-assemblies, such as the double crossover (DX) $[4,5]$, the triple crossover (TX) $[6,7]$, the paranemic crossover (PX) [8-10], $4 \times 4$ tile [11]. Many of them have been used as scaffolds for protein and NP arrays [1, 2, 12-14]. In particular, Yan et al. have successfully obtained gold NPs matrix scaffolded by DNA lattice $[12,13]$.
Here we report our results of $1 \mathrm{D}$ and $2 \mathrm{D}$ arrays of gold NPs using DNA arrays as scaffolds. The $4 \times 4$ tile, as the building block of the arrays, is modified from the one developed by Yan et al. [11]. One of the component strands of the motif was biotin modified, so it had a strong affinity to streptavidin modified $5 \mathrm{~nm}$ gold NP. By mixing the DNA array with the streptavidin modified gold NPs, the metallic array was formed on the DNA template. The $4 \times 4$ array was examined by atomic force microscopy (AFM) and the metallic arrays were observed by transmission electron microscope (TEM).

\section{MATERIALS AND EXPERIMENTAL}

Custom DNA strands, for the $4 \times 4$ tiles (sequence design shown in Figure 1), were purchased from Integrated DNA Technology (Leuven, Belgium). Strands were purified by electrophoresis; bands were tailored out of $15 \%$ denaturing polyacrylamide gels and eluted in a solution containing $500 \mathrm{~mm}$ ammonium acetate, $10 \mathrm{~mm}$ magnesium acetate, and $1 \mathrm{~mm}$ EDTA. Five nm gold NP labeled with streptavidin solution is purchased from Sigma Aldrich (Mo, USA).

DNA arrays were formed by mixing a stoichiometric quantity of corresponding strands, quantified by $\mathrm{OD}_{260}$, in $1 \times \mathrm{TAE} / \mathrm{Mg}\left(20 \mathrm{~mm}\right.$ Tris, $2 \mathrm{~mm}$ EDTA, and $\left.12.5 \mathrm{~mm} \mathrm{MgCl}_{2}\right)$ buffer. The final concentration of each DNA strand was 


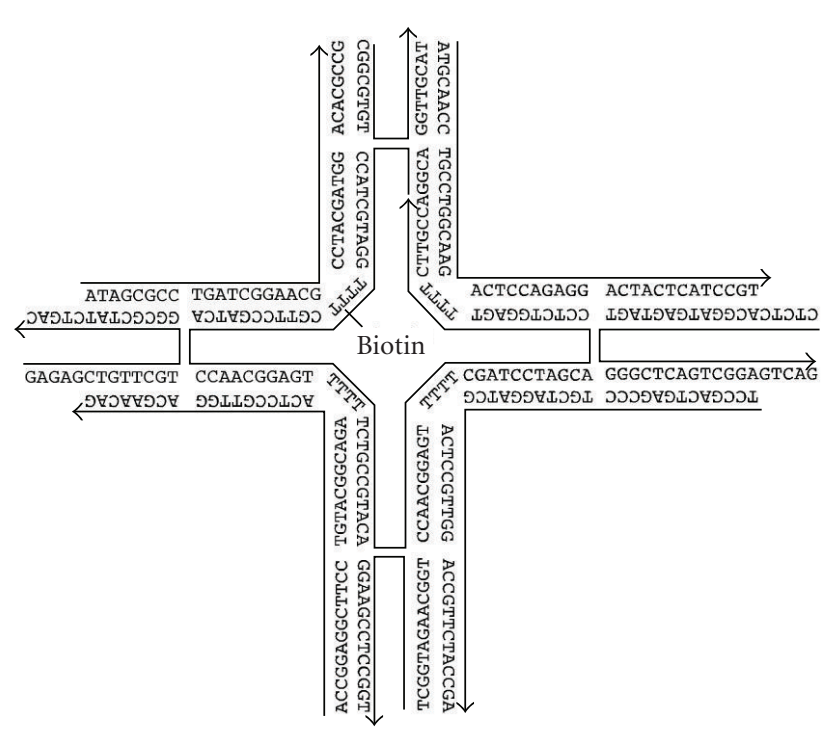

(a)

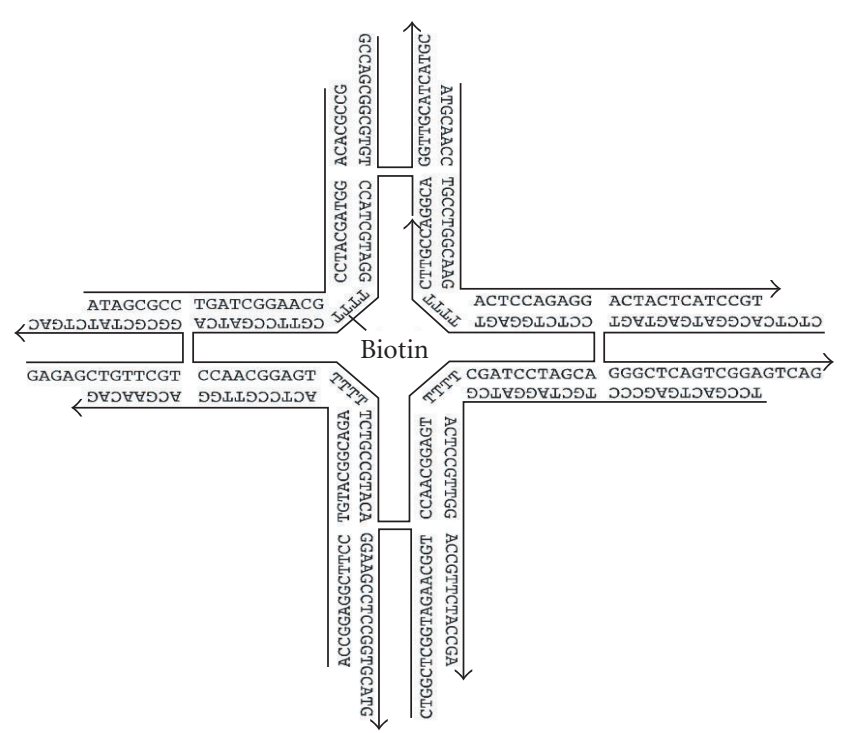

(b)

Figure 1: The sequence design of the $4 \times 4$ tiles. (a) The $4 \times 4$ tile motif of the DNA template for the $1 \mathrm{D}$ gold NP array. (b) The $4 \times 4$ tile motif of the DNA template for the 2D gold NP array.

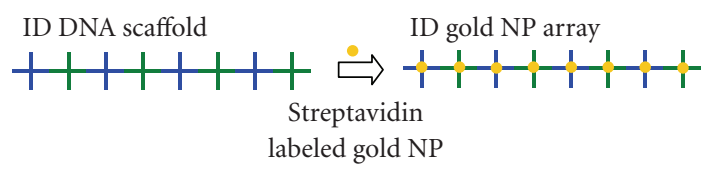

(a)

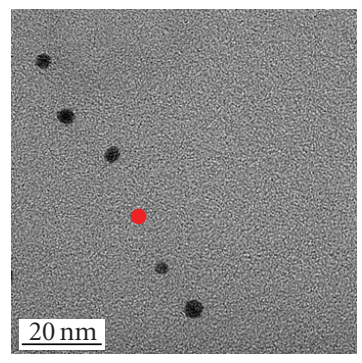

(b)

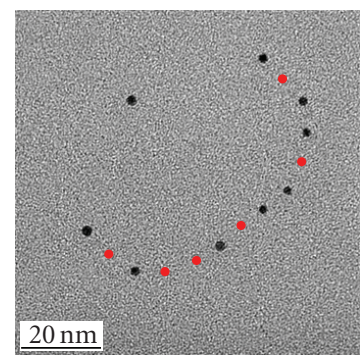

(c)

FIGURE 2: The 1D DNA template and the derivative 1D gold NP array. (a) The pathway of 1D gold NP array from the 1D DNA template. The yellow dots denote streptavidin labeled NPs, which were able to be anchored at the center of each $4 \times 4$ tile by the binding between biotin and streptavidin. (b), (c) The TEM image of the 1D gold NP array. The scale bars in both (b) and (c) are $20 \mathrm{~nm}$.

$1.0 \mu \mathrm{M}$ and the final volume was $50 \mu \mathrm{L}$. DNA strand mixtures were cooled slowly from $95^{\circ} \mathrm{C}$ to $4^{\circ} \mathrm{C}$ in a heating block for 51 hours to facilitate hybridization.

A small drop of the DNA lattice sample $(2 \mu \mathrm{L})$ was deposited on the freshly cleaved mica surface. $1 \times \mathrm{TAE} / \mathrm{Mg}$ buffer $(400 \mu \mathrm{L})$ was then added to the drop on the mica. The DNA lattice was imaged in a fluid cell in semicontact mode on a Ntegra Aura atomic force microscope (NT-MDT Co., Moscow, Russia) using MSCT/Au tips (Veeco Inc., NY, USA).

The hybridized DNA scaffold sample was mixed with $5 \mathrm{~nm}$ gold NPs labeled with streptavidin overnight. TEM images were obtained with a Jeol JEM 2010 microscope. A holey carbon film coated copper grid was placed floating on a $5 \mu \mathrm{L}$ droplet of the mixture of the DNA scaffold and the NPs for 5 minutes. The excess liquid was then removed from the grid by lightly touching the edge of a filter paper. The sample grid was put in a desiccator overnight, before the TEM examination.

\section{RESULTS AND DISCUSSION}

\subsection{D gold NP array}

The $4 \times 4$ DNA tile, modified from the motif developed by Yan et al. [11], was used to build the 1D scaffold. The sequence of the $4 \times 4$ tile motif with nine strands is shown in Figure 1(a). The modified sticky end matching enabled motifs to form a ribbon-like 1D DNA scaffold as shown in Figure 2(a). The 1D scaffold was used to obtain the 1D gold NP array after adding the streptavidin labeled gold NPs as shown in Figure 2(a). The strand mixture in TAE/Mg buffer was slowly cooled down to facilitate the self-assembly process. Referring to Figure 1(a), there was a biotin group grafted on one of the TTTT segments of the center strand, so the motif was able to capture the streptavidin labeled $5 \mathrm{~nm}$ gold NP. By mixing streptavidin labeled gold NPs with the $4 \times 4$ tile array in the buffer solution, the gold NP array was then obtained (as shown schematically in Figure 2(a)). 

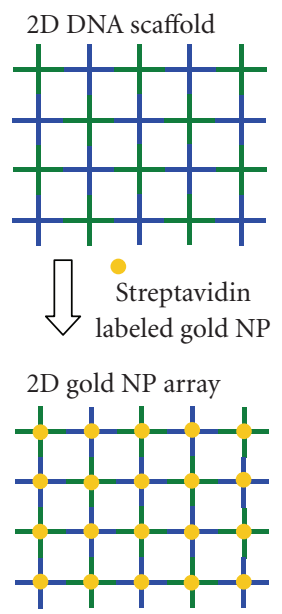

(a)

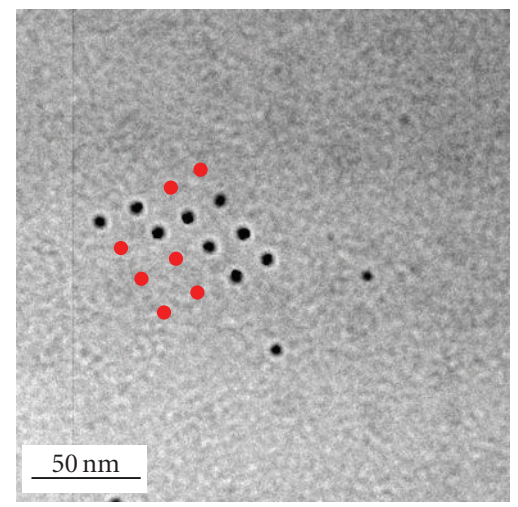

(c)

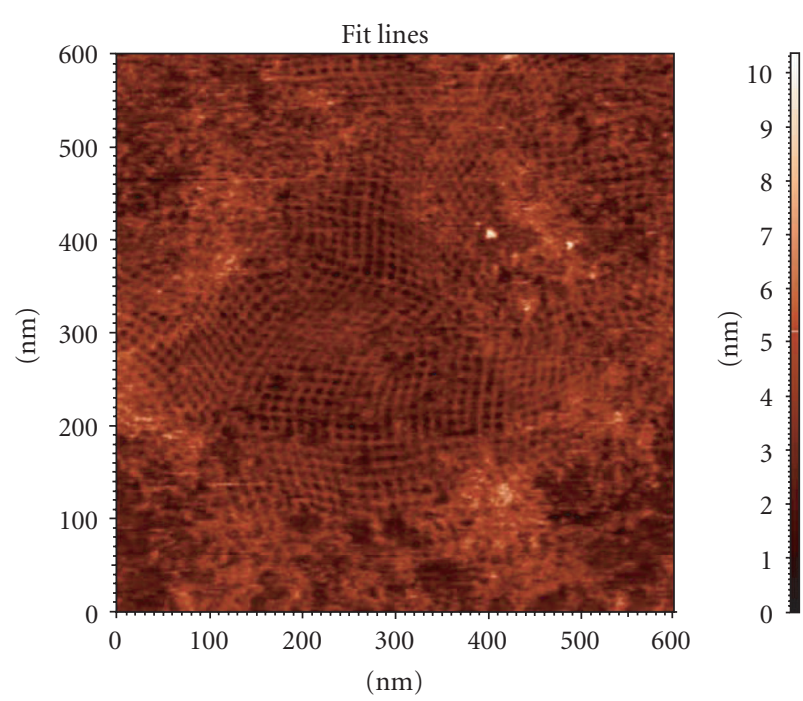

(b)

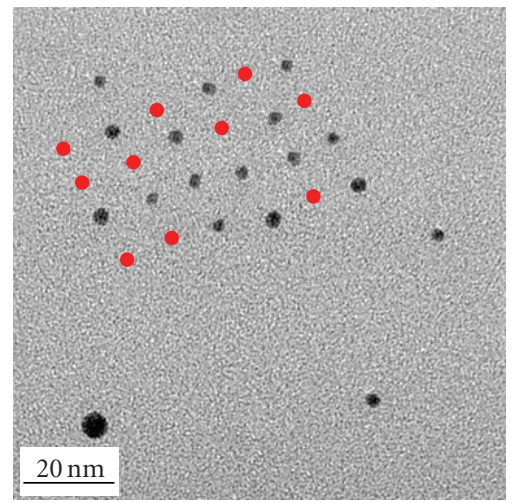

(d)

FIGURE 3: The 2D DNA template and the derivative 2D gold NP array. (a) The pathway of 2D gold NP array from the 2D DNA scaffold. The yellow dots denote streptavidin labeled NPs to be anchored at the center of each $4 \times 4$ tile motif by the binding between biotin and streptavidin. (b) The AFM image of the 2D DNA scaffold. (c), (d) The TEM images of the 2D gold NP arrays. The scale bar of (c) is $50 \mathrm{~nm}$ and the scale bar of $(\mathrm{d})$ is $20 \mathrm{~nm}$.

According to the TEM images (Figures 2(b) and 2(c)), the gold NPs were $5.0 \pm 0.8 \mathrm{~nm}$ in diameter and the center-tocenter distances of the gold NPs were in a range of 15.8 to $19.4 \mathrm{~nm}$, which is within the design parameter of the DNA template. The red dots in Figures 2(b) and 2(c) represent the missing NPs due to the imperfect binding efficiency. The future task of such research is on how to enhance the binding efficiency in order to achieve more perfect NP arrays in larger scale.

\subsection{D gold NP array}

The 2D gold NP array was obtained by the pathway strategically similar to that of the $1 \mathrm{D} \mathrm{NP}$ array. The $4 \times 4$ tile motif, shown in Figure 1(b), with chosen lattice-forming sticky end matching strategy, was used to form the 2D DNA scaffold for positioning gold NPs in a 2D array. The 2D DNA scaffold was formed in the cooling down process and was examined by AFM in liquid. The streptavidin labeled gold NPs were mixed with the 2D DNA scaffold in the buffer solution to form the 2D gold NP array as shown in Figure 3(a). The AFM image of the 2D DNA scaffold is shown in Figure 3(b). The 2D gold NP array was examined by TEM. According to the TEM images (Figures 3(c) and 3(d)), the gold NPs were $5.0 \pm 0.8 \mathrm{~nm}$ in diameter and the center-to-center distances of the gold NPs were in a range of 15.4 to $18.6 \mathrm{~nm}$, which is within the design parameter of the 2D DNA template. Again, the red dots in Figures 3(c) and 3(d) represent the missing NPs due to the imperfect binding efficiency. Although Figures 3(c) and 3(d) are not perfect arrays of gold NPs, this study shows that such DNA scaffolding technique has the potential to position gold NPs in nanoscale rather precisely. Our future study will be focused on developing DNA scaffolds in larger scale and enhancing better binding between DNA scaffolds and the gold NPs. We are also trying to bind the magnetic NPs on the DNA scaffolds, which should have applications in information storage technology. 


\section{CONCLUSION}

In summary, we have successfully applied self-assembled DNA scaffold to pattern $5 \mathrm{~nm}$ gold NPs into the $1 \mathrm{D}$ and the $2 \mathrm{D}$ arrays. This study shows that it is possible to engineer a desired NP pattern by designing a specific DNA scaffold (e.g., pattern, spacing, and size). However, the efficiency of the binding between the scaffold and NPs is still not high enough. It is difficult to obtain NP arrays in larger scale insofar. The popular methods for NP and DNA binding are based on either DNA base pairing or streptavidin-biotin binding, yet both bindings are not strong enough. Therefore, it is desirable to find another stronger binding mechanism for NPs to be anchored on DNA scaffolds. It is still quite intriguing to obtain well-patterned NP arrays with the simple method of DNA nanotechnology. When DNA arrays can be more controllable and the binding efficiency of the gold NPs on the DNA scaffold is further enhanced, this DNA nanotechnology methodology will be powerful to scaffold NPs into nanoscaled devices.

\section{ACKNOWLEDGMENT}

The authors greatly appreciate the financial support of the RGC HKUST 602405 of the earmarked grant from the University Grant Council of the Hong Kong government. The project is entitled: "The preparation of nano-magnetic supermatrix templated by DNA self-assembly."

\section{REFERENCES}

[1] C. A. Mirkin, R. L. Letsinger, R. C. Mucic, and J. J. Storhoff, "A DNA-based method for rationally assembling nanoparticles into macroscopic materials," Nature, vol. 382, no. 6592, pp. 607-609, 1996.

[2] D. Zanchet, C. M. Micheel, W. J. Parak, D. Gerion, and A. Paul Alivisatos, "Electrophoretic isolation of discrete Au nanocrystal/DNA conjugates," Nano Letters, vol. 1, no. 1, pp. 32-35, 2001.

[3] B. H. Robinson and N. C. Seeman, "The design of a biochip: a self-assembling molecular-scale memory device," Protein Engineering, vol. 1, no. 4, pp. 295-300, 1987.

[4] X. Li, X. Yang, J. Qi, and N. C. Seeman, "Antiparallel DNA double crossover molecules as components for nanoconstruction," Journal of the American Chemical Society, vol. 118, no. 26, pp. 6131-6140, 1996.

[5] E. Winfree, F. Liu, L. A. Wenzler, and N. C. Seeman, "Design and self-assembly of two-dimensional DNA crystals," Nature, vol. 394, no. 6693, pp. 539-544, 1998.

[6] T. H. LaBean, H. Yan, J. Kopatsch, et al., "Construction, analysis, ligation, and self-assembly of DNA triple crossover complexes," Journal of the American Chemical Society, vol. 122, no. 9, pp. 1848-1860, 2000.

[7] D. Liu, S. H. Park, J. H. Reif, and T. H. LaBean, "DNA nanotubes self-assembled from triple-crossover tiles as templates for conductive nanowires," Proceedings of the National Academy of Sciences of the United States of America, vol. 101, no. 3, pp. 717-722, 2004.

[8] N. C. Seeman, "DNA nicks and nodes and nanotechnology," Nano Letters, vol. 1, no. 1, pp. 22-26, 2001.

[9] X. Zhang, H. Yan, Z. Shen, and N. C. Seeman, "Paranemic cohesion of topologically-closed DNA molecules," Journal of the
American Chemical Society, vol. 124, no. 44, pp. 12940-12941, 2002.

[10] Z. Shen, H. Yan, T. Wang, and N. C. Seeman, "Paranemic crossover DNA: a generalized holliday structure with applications in nanotechnology," Journal of the American Chemical Society, vol. 126, no. 6, pp. 1666-1674, 2004.

[11] H. Yan, S. H. Park, G. Finkelstein, J. H. Reif, and T. H. LaBean, "DNA-templated self-assembly of protein arrays and highly conductive nanowires," Science, vol. 301, no. 5641, pp. 1882$1884,2003$.

[12] J. Zhang, Y. Liu, Y. Ke, and H. Yan, "Periodic square-like gold nanoparticle arrays templated by self-assembled 2D DNA nanogrids on a surface," Nano Letters, vol. 6, no. 2, pp. 248251, 2006.

[13] J. Sharma, R. Chhabra, Y. Liu, Y. Ke, and H. Yan, "DNAtemplated self-assembly of two-dimensional and periodical gold nanoparticle arrays," Angewandte Chemie International Edition, vol. 45, no. 5, pp. 730-735, 2006.

[14] H. Li, S. H. Park, J. H. Reif, T. H. LaBean, and H. Yan, "DNAtemplated self-assembly of protein and nanoparticle linear arrays," Journal of the American Chemical Society, vol. 126, no. 2, pp. 418-419, 2004. 

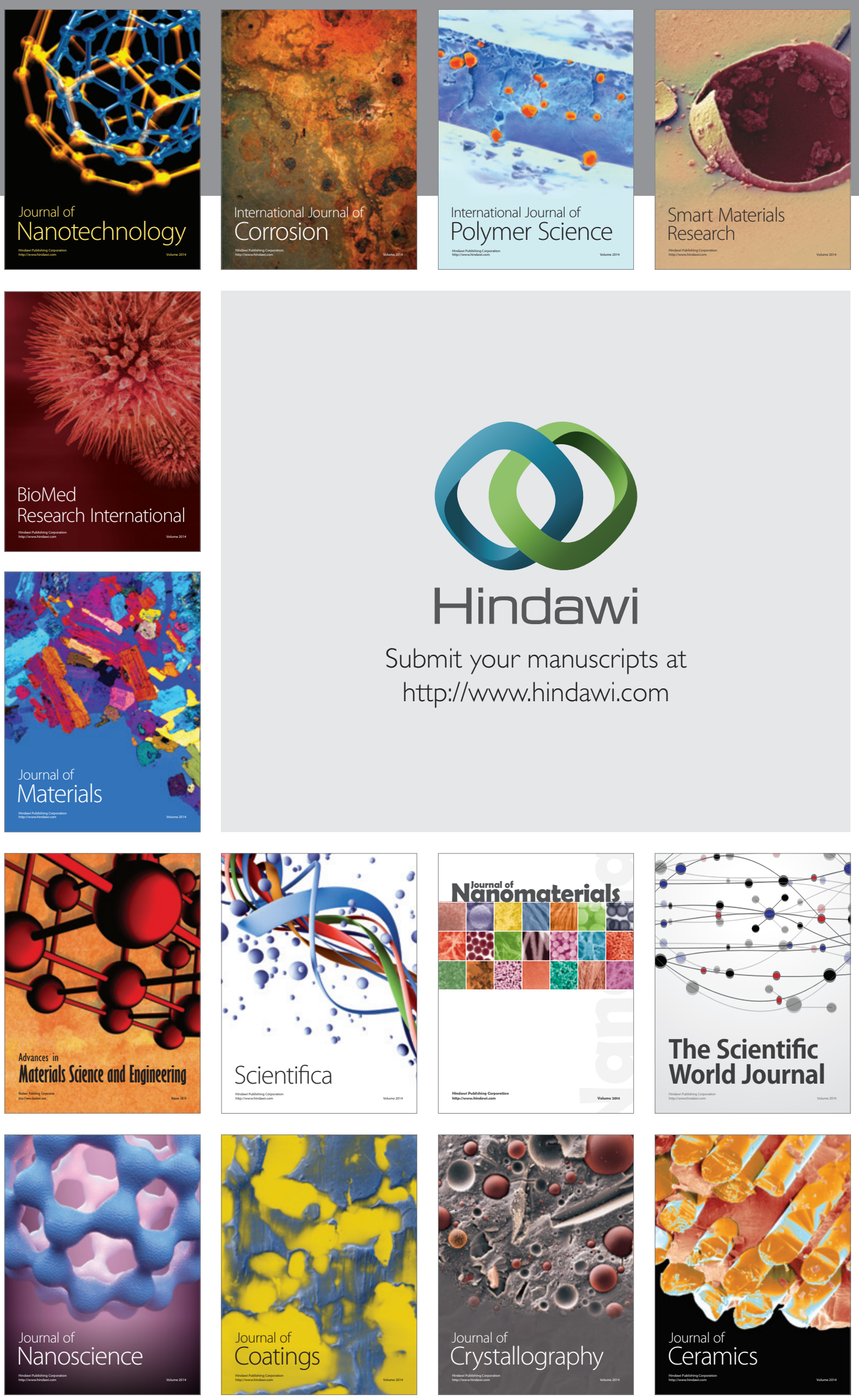

The Scientific World Journal

Submit your manuscripts at

http://www.hindawi.com

\section{World Journal}

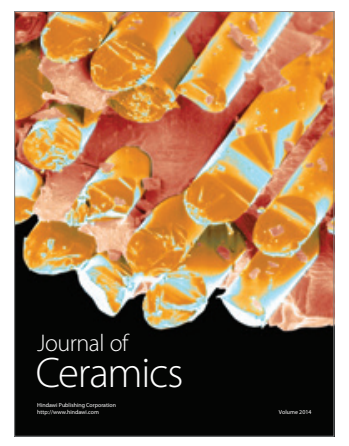

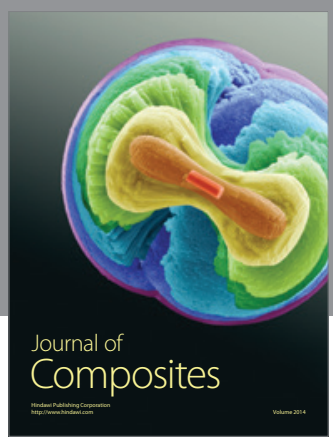
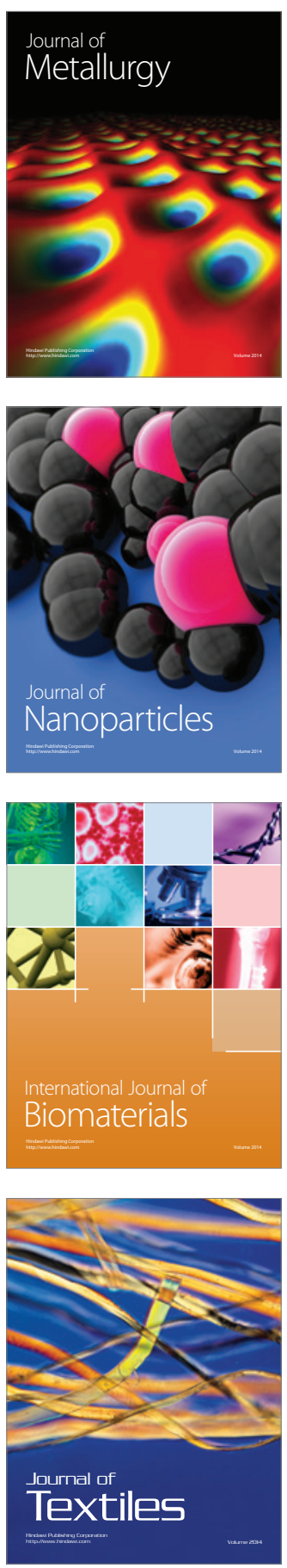